\title{
EFFECT OF XARELTO ON HEMORHEOLOGICAL SYSTEM IN PATIENT GROUP WITH PERMANENT FORM OF ATRIAL FIBRILLATION
}

\author{
${ }^{1}$ PhD student Maia Gotsadze, ${ }^{1}$ Professor Nugzar Narsia, \\ ${ }^{2}$ PhD Nana Momtseldze, ${ }^{2}$ Professor Maia Mantskava \\ ${ }^{1}$ Tbilisi State Medical University, Georgia, Tbilisi \\ ${ }^{2}$ I. Beritashvili Center of Experimental Biomedicine, Georgia, Tbilisi \\ ${ }^{3}$ St. Lazare Clinic, Georgia, Tbilisi
}

DOI: https://doi.org/10.31435/rsglobal_ws/30112019/6770

\section{ARTICLE INFO}

Received: 21 September 2019

Accepted: 10 November 2019

Published: 30 November 2019

\section{KEYWORDS}

Rhythm disorder,

Erythrocytes aggregation,

Anticoagulant.

\begin{abstract}
Atrial fibrillation and its associated complications, such as ischemic stroke and systemic thromboembolism, remain as an urgent problem of today's medicine. For the patients who are diagnosed by atrial fibrillation, an anticoagulant drug therapy is necessary. The main goal of our research was to study the rheological parameters in patients diagnosed with a permanent form of arrhythmia, namely to evaluated erythrocytes aggregation, eritrocytes membranes deformiability, plasma viscosity. In addition, we were interested in the effect of a direct inhibitor of micronized factor Xa - Xarelto on hemorheology to determine which hemorheologycal parameters play a primary role in the regulation of blood flow and how are provided the therapeutical effects of the medicine.
\end{abstract}

Citation: Maia Gotsadze, Nugzar Narsia, Nana Momtseldze, Maia Mantskava. (2019) Effect of Xarelto on Hemorheological System in Patient Group with Permanent form of Atrial Fibrillation. World Science. 11(51), Vol.2. doi: 10.31435/rsglobal_ws/30112019/6770

Copyright: (C) 2019 Maia Gotsadze, Nugzar Narsia, Nana Momtseldze, Maia Mantskava. This is an openaccess article distributed under the terms of the Creative Commons Attribution License (CC BY). The use, distribution or reproduction in other forums is permitted, provided the original author(s) or licensor are credited and that the original publication in this journal is cited, in accordance with accepted academic practice. No use, distribution or reproduction is permitted which does not comply with these terms.

Introduction. The prevalence of atrial fibrillation, associated morbidity, and mortality is increasing year by year worldwide [1]. Currently, more than 6 million people in Europe are suffering from atrial fibrillation. Over the next 50 years, along with an increase in the average age of the population, the frequency of spreading the disease will increase by about 2.5 times [2]. Atrial fibrillation can be called a non-infectious epidemic of our time. Lack of coordination of atrial contractions contributes to blood coagulation, the formation of blood clots, which leads to the creation of an embolism. Therefore, against the background of this rhythm disorder, it is common to develop ischemic strokes and systemic thromboembolism. Dr. Steve Roach-Professor of Neurology and Director of the Comprehensive Epilepsy Program at wake Forest University medical School wrote, that: "What prevents blood flow and causes stagnation of blood flow, contributes to the occurrence of thrombosis and, consequently, stroke." [3]. Atrial fibrillation has several forms: paroxysmal, persistent, permanent forms. And, undoubtedly, all of them significantly worsen the quality of human life, cause health problems, provoke the occurrence of such harmful diseases as cerebral and myocardial infarctions, and so on. The risk of systemic thromboembolic conditions in all categories of patients sharply increases 48 hours after the onset of arrhythmia, which requires antithrombotic and anticoagulant therapy. In this regard, randomized clinical trials are conducted permanently: PIAF (Pharmacological Intervention in Atrial Fibrillation), AFFIRM (Atrial Fibrillation Follow-Up Investigation of Rhythm Management), STAF (Strategies of Treatment of Atrial Fibrillation), HOT CAFÉ (How to Treat Chronic Atrial Fibrillation). The XANTUS (Xarelto for Prevention of Stroke in Patients with Atrial Fibrillation). RE-LY (Randomized Evaluation of Long-Term Anticoagulant Therapy). 
Based on the standard strategy for the management and treatment of patients with arrhythmia, it can be concluded that antithrombotic therapy is carried out against the background of monitoring the coagulation system, while the rheological state of blood is considered neither during the initial diagnosis of disease nor in the implementation of preventive measures. In the treatment of atrial fibrillation, the rheological system is not considered as the target of therapy, although several scientific works are confirming that abnormal blood rheology is a risk- factor for the development of many cardiological pathologies [4,5].The fact that anticoagulation therapy is directed to the bloodstream to promote blood flow does not require any specific evidence. However, blood flow as a pathophysiological parameter is not based solely on platelet and fibrinogen variables; But on the blood flow also plays a role in the membranes of other blood cells and their aggregating/disaggregating ability. Therefore, regardless of whether coagulation is in order, there is always a risk of capillary stasis, which is also crucial for blood circulation. Thus, blood flow is the result of two parallel, indirectly interconnected, yet different systems (hemorheological and coagulation/anticoagulation systems). It should be noted that during normal processes, coagulation and anticoagulation are in a balanced state, although even with the physiological states, the exhaustion of the anticoagulant system is more intense than the coagulation system. It is like this because of the use of an almost universal practice of anticoagulant therapy is based, which, of course, is especially important for preventive measures. However, such an approach comes with the controversial issues [6]. The rheological system in the body does not have counterbalanced reactions that, like anti rheological effects, would ensure the release of red blood cells from aggregates and conglomerates. Based on this, the rheological system is a very valuable system, and it is essential to maintain it. Violation of hemorheological properties leads to the formation of a physical substance (stagnant area, instantly aggregated erythrocyte accumulation).

Anticoagulant therapy (GMN) is an essential link in the treatment of patients with atrial fibrillation. By the 2016 ESC Guidelines for the management of atrial fibrillation developed in collaboration with EACTS and by the 2014 AHA/ACC/HRS Guideline for the Management of Patients with Atrial Fibrillation and A Report of the American College of Cardiology/American Heart Association Task Force on Practice Guidelines and the Heart Rhythm Society in patients with different forms of non-valvular genesis AF is indicated-NOACs. All NOACs have a predictable effect (onset and offset) without the need for regular anticoagulation monitoring. However, their impact on rheological parameters has not been thoroughly studied to date. For the first time, we evaluated the effect on hemorheology of Xarelto (Rivaroxaban (Bayer Schering Pharma, $20 \mathrm{mg}$ pills) the direct inhibitor of micronized Xa factor for stroke and systemic thromboembolism prophylaxis in nonvalvular atrial fibrillation). In this article, we will discuss the effect of NOAK - Xarelto on hemorheological parameters during the permanent form of arrhythmia. Such an approach is scientific news. Although Xarelto, according to the results of clinical expertise as a result of pharmacy -marketing analysis, is considered to be a better acting safe drug, hemorheological clinical trials were not included in the protocols at the time of entering the market. There is no doubt that the results of our study are very relevant since they shed light on hitherto unknown hemorheological reactions under the influence of Xarelto.

The aim of the research. The purpose of the present study was to find out the differences in the cohort of patients with permanent form of arrhythmia and in a cohort of non-arrhythmic individuals of similar age and to assess the effect of a new generation oral anticoagulant-Xarelto on a hemorheological parameters in a group of patients with a permanent form of arrhythmia, namely on erythrocytes aggregability, their membrane deformability and hematocrit.

Research Materials and Methods: In Tbilisi St. Lazarus clinic we examined by the 12 patients (women:men $-3: 1$, age of $65 \pm 7,2$ year ). The following studies: Clinical studies: ECG, EQO to diagnostic the forms of rhythm disorders, index of erythrocytes aggregability, deformability, plasma viscosity to evaluate the blood rheological parameter; Coagulogramm to determine of coagulation condition. Studies have been made in the Department of Rheological and Diagnostic-Analytical Services of LEPL Beritaishvili Center for Experimental Biomedicine and NRAP Society of Rheology. The methods: Hemodynamic parameters were checked in those patients. The following was done: ECG with cardiograph NIHON KONDEV cardiofax GEM (Japanese); Echocardiography with PHILIPS clearVue 550 (Netherlands).

Hemorheological research was done with the newest methodologies: The study of Erythrocytes aggregation, Erythrocytes deformation and plasma viscosity can only be done in the Department of Rheology and Diagnostic-Analytical Services of I.Beritashvili Center of Experimental 
Biomedicine. The Index of the Erythrocytes aggregation is the area of aggregated erythrocytes divided by the full area of the erythrocytes. This can be done with the system of textural analyzes (Tas-Plus, Leitz, Germany). These new innovative methods "Georgian Technique" is created by Georgian scientists and they are famous in the world as direct, numeral and exact $[7,8]$. The index of the deformation of the erythrocytes (nucleopore membrane filter method) [9] is done with filtration method. Plasma viscosity is measured in the capillary viscometer in $37^{\circ} \mathrm{C}$. The movement of plasma in the capillaries is induced by the force of gravity.

Necessary studies which were given by the Ministry of Labor Health and Social Affairs of Georgia:Clinical analyses of blood (WBC, RBC, HGB, HCT, PLT, ROE)(With Humancounts (Germany)); Coagulogram(PT Prothrombin Time, APTT Activated Partial Thromboplastin Time TT Thrombin Time, FIB Fibrinogen, Fa-I Factor Intrinsic, Fa-E Factor Extrinsic Selectable dimensions, Seconds, INR, \%, U (mg/d) Coatron M1 (Germany)).

Target group: The patients with permanent form of arrhythmias. N-12. They received Xarelto 20 $\mathrm{mg}$ daily for 3 week. The study was conducted in clinical-laboratory direction. The database was statistically processed with the special statistical programs of the biological system: SPSS which demonstrated.

Results. From statistical analysis, we found that erythrocyte aggregability during the permanent form of atrial fibrillation was higher than almost 50\% in the control group. Membrane deformability was also different between the control group and in the patients' group with arrhythmia. A similar tendency was found for plasma viscosity, ie plasma viscosity increased during atrial fibrillation compared to controls. It should also be noted that the results of the hemodynamics and coagulation monitoring process are in full compliance with the standards found in atrial fibrillations. To achieve the ultimate goal of the study, we examined the full spectrum of hemorheological parameters before and after the treatment of Xarelto in a permanent form of arrhythmias. For detailed information, see the table.

Table.1. Index of Erythrocytes aggregation, deformability, plasma viscosity in patients group with a permanent form of atrial fibrillation before and after treatment with Xarelto and the same parameters in the control group, $\mathrm{M} \pm \mathrm{m}$

\begin{tabular}{|l|l|l|l|l|}
\hline \multicolumn{2}{|c|}{} & \multicolumn{1}{|c|}{ EAI, \% } & EDI, \% & \multicolumn{1}{c|}{ VpI plasma, sP } \\
\hline $\begin{array}{l}\text { Permanent } \\
\text { form of AF }\end{array}$ & $\begin{array}{l}\text { Before } \\
\text { treatment }\end{array}$ & $38,07 \pm 5,98$ & $2,33 \pm 0,14$ & $1,15 \pm 0,09$ \\
\cline { 2 - 5 } & $\begin{array}{l}\text { After } \\
\text { treatment }\end{array}$ & $35,64 \pm 4,83$ & $2,12 \pm 0,29$ & $1,11 \pm 0,01$ \\
\hline Control & $25,61 \pm 1,29$ & $2,09 \pm 0,25$ & $1,10 \pm 0,35$ \\
\hline
\end{tabular}

Assessment of the complete spectra revealed that hemorheological changes were observed in a group of patients with a permanent form of atrial fibrillation. A clear expression of the changes was loaded by the erythrocyte aggregability, which clearly demonstrates the disruption of blood flow, and is confirmed in the modern scientific literature $[7,8]$.

As for erythrocyte membrane deformability, although the analytical change is not volumetric (the numbers are not very different and only increase by $5 \%$ ), this change is sufficient to increase erythrocyte hardness and impede normal blood flow to the myocardial microcirculation. Plasma viscosity was expected to increase, however, so far, no difference of plasma viscosity between forms has been reported in the literature [10].

It is also very interesting to note that in the patient groups, which received Xarelto the improvement of hemorheological features was observed, and their normalization was in all parameters of Rheology. Here too, as noted above, was the most marked improvement in the aggregation mechanism. Probably, this is the leading link in terms of blood flow pathology. How much the erythrocyte aggregability is depended on the membrane's deformability and plasma viscosity is not unequivocal. It is difficult to allow the plasma viscosity and blood viscosity to be the same magnitude when we are talking about blood circulation [11]. It is also difficult to agree that aggregability is an entirely independent fraction of blood flow [12]. 
Conclusions. In our opinion, the positive impact of Xarelto also depends on the fact that a small percentage increase in viscosity during atrial fibrillation is fully regulated with Xarelto and is, in fact, equal to the control units. All of this not only points to the positive effect of Xarelto on the hemorheological system during arrhythmia but also helps us to study the mechanisms underlying the abnormal blood flow in arrhythmia. It is essential to note that our research was conducted in the background of a common standard, in particular, monitoring of the coagulation system and hemodynamics. There is no doubt the positive effect of Xarelto on coagulation. But equally important are the results of our study, which highlighted the fact that positive changes in the body depend not only on the anticoagulant system saturated, which is possible by other anticoagulants, but also were solved the problem of blood flow, and this is most important to avoid the stasis. If we take into account that such work is performed for the first time, in which has been studied impact of Xarelto on blood rheology and coagulation parameters in the background of hemodynamic monitoring, it can be said that the results of research, are scientific innovation, allowing us to pay attention to the blood rheology parameters as a diagnostic a treatment target. This is important for disease prevention, treatment optimization, and evaluation of prognosis.

There is no doubt the need for intensive growth of the study array, incorporating other forms of atrial fibrillation with similar protocols into the aims and objectives of the study.

Extensive scientific work and clinical research are vital for the study of the pathophysiology of arrhythmia, to choose the right disease management strategy. This is one of the interesting and topical issues of biomedical direction.

\section{REFERENCES}

1. https://www.uptodate.com/contents/epidemiology-of-and-risk-factors-for-atrial-fibrillation

2. Zulkifly H, Lip GYH, Lane DA. Epidemiology of atrial fibrillation. Int J Clin Pract. 2018 Mar;72(3):e13070. doi: 10.1111/ijcp.13070.

3. https://dellmed.utexas.edu/directory/steve-roach

4. Gordon Lowe, Ann Rumley, John Norrie, Ian Ford, James Shepherd, Stuart Cobbe, Peter Macfarlane, Christopher Packard. Blood Rheology, Cardiovascular Risk Factors, and Cardiovascular Disease: The West of Scotland Coronary Prevention Study J. Thromb Haemost 2000; 84: 553-8

5. https://clinicaltrials.gov/ct2/show/NCT03506984

6. Hermosillo A.J., Spinler S.A. Aspirin, Clopidogrel, and Warfarin: Is the Combination Appropriate and Effective or Inappropriate and Too Dangerous? Ann Pharmacother 2008; 42 (6): 790-805.

7. G.Mchedlishvili Hemorheological changes in microcirculation: their mechanism and measurement technique. Indian J Exp Biol. 2007 Jan;45(1):32-40. Review.

8. M.Mantskava. New and Newest approaches for measurements of blood flow. M.Nauka, 189, p. 2019

9. Titovsky, A.B. Measurement of the deformability of erythrocytes in a stream under the influence of gravitational forces / A.B. Titovsky // Materials of the Intern. microcirculation conferences. -M.: Yaroslavl, 2019.S. 205-206

10. Blagova O.V. Drad treatment atrial fibrillation. M.: BookMedMedia, 2019. $148 \mathrm{~s}$.

11. Dan G.-A., de Luna A.B., CammJ. Atrial fibrillation therapy. London: SpringerVerlag, 2014. 188 p

12. Muravyov A.V., Kislov N.V., Tikhomirova I.A., Mikhailov P.V., Muravyov A.A. Influence of plasma viscosity and hematocrit on erythrocytes deformability // Russian Journal of Biomechanics. $2013 . \quad$ No2. https://cyberleninka.ru/article/n/vliyanie-vyazkosti-plazmy-i-gematokrita-na-deformatsiyu-eritrotsitov (11/14/2019). 